"This is the peer reviewed version of the following article:

A layered beam element for modeling de-bonding of steel bars in concrete and its detection using static measurements

which has been published in final form at

https://onlinelibrary.wiley.com/doi/abs/10.1002/stc.2142

This article may be used for non-commercial purposes in accordance with Wiley Terms and Conditions for Self-Archiving 


\title{
A Layered Beam Element for Modelling De-bonding of Steel Bars in Concrete and its Detection using Static Measurements
}

\author{
by \\ K. Liu ${ }^{1}$, S.S. Law $^{2}$ and X.Q. Zhu*3
}

\begin{abstract}
In the formulation of finite elements, the variation of elemental internal forces and displacements are interpolated. The force interpolation functions are known to reproduce the variations of forces better than the interpolation functions on the displacements. Layered section beam model is not as complicated as the fiber model and yet it is much more accurate than ordinary beam model. The force-based finite element is revisited in this paper with its application in the numerical studies of a damage detection strategy for a reinforced concrete beam under static load. Two kinds of damages are studied including the cracking or other local damage of the concrete and the bonding between the concrete and the steel bar. Both kind of damages in an element can be detected separately or in combinations with the proposed strategy. The force-based layered finite element is shown to be a practical, accurate and efficient representation of the bonding damage of steel bars in concrete structures.
\end{abstract}

Keywords: Damage; reinforced concrete; layered section model; force-based; de-bonding detection.

\footnotetext{
${ }^{1}$ Lecturer, School of Civil Engineering, Harbin Institute of Technology.

${ }^{2}$ Professor, School of Civil Engineering, Chongqing University

3 Corresponding author. Associate Professor, School of Civil and Environmental Engineering, University of Technology Sydney, NSW, Australia. Email: xinqun.zhu@uts.edu.au
} 


\section{Introduction}

The damage identification of structures has drawn active attention from various engineering fields in recent years. Existing approaches in this area can be classified into two major categories, i.e. the dynamic identification methods and the static identification methods. The dynamic method has been developed more maturely as reported in Zimmeman and Smith [1], Doebling et al. [2] and Salawu [3]. Research topics have been highlighted for further development into practical methods for damage identification, such as the mass change and damping variation associated with the local damage and the need to remove the noise effect in the measured higher vibration modes.

Banan and Hjelmstad [4,5] have proposed a recursive quadratic programming method for the parameter estimation of structures with measured displacements from static loads. This approach has received considerable attention from many researchers (Di Paola and Bilello [8], Wang et al.[7], Liu and Chian [6]). The authors have also contributed to further development of this approach (Zhu et al.[10], Zhu and Law[9]).

Common defects with reinforced concrete structure are cracking and spalling as visualized in the inspection of the structural components. However, the bonding between concrete and steel bars is, in fact, the most influential factor for different kinds of damages, and it can be taken as the primary indicator on any damage with a structural component. Such bonding has been investigated widely with the development of several models [11-15]. All studies showed that the load-carrying capacity of the structure will drop dramatically when de-bonding occurs. Many research have been conducted on the detection of local defects at the interface of concrete structures [16-18]. There are also a few reports of global approaches on debonding detection. Zhu and Law [9] developed a displacement-based interface finite element for detecting the de-bonding of steel bars in concrete structures using static test data. However, multiple damage in concrete and debonding have not been investigated which may probably due to the complexity of the beam model and method of damage detection. Recently, Biswal and Ramaswamy [19] considered the uncertainties in measurements using interval bounds and the damage index with interval parameters was used to quantify the damage.

The forced-based finite element and the displacement-based finite elements are two numerical models of structures. The latter is commonly used with the development of computation facilities. However, the former has been shown to have better accuracy in the representation of the variation of the internal forces compared to the latter which is based on the displacement interpolation functions. Force-based finite element model for the bond slip analysis of the concrete structures has been proposed by Salari and Spacone [20,21]. Since the contribution of the bonding stiffness to the 
global stiffness of the beam element is very small, a refined model is needed to represent this contribution accurately and properly such that the identification in the inverse problem can be conducted more effectively and appropriately. However, no work has been done in this aspect for damage identification of structures.

This paper attempts to improve the accuracy and robustness of de-bonding identification of concrete structures using the force-based finite element with the development of an identification strategy and a layered finite element. The proposed method detects both local damage and debonding in a beam element simultaneously. Results shown indicate that both single and multiple damages can be effectively identified from noisy static measurements with the proposed strategy. The system identification method is based on the existence of debonded segment in reinforcement and a cracked region in the concrete beam to get the force-deformation relationship. The latter is then used for the damage detection. This is different from the usual approach with stress analysis starting with the constitutive equation of material and the stress-strain relationship to trace the development of concrete cracks and debonding in reinforcement. The stress-strain relationship such as that on the transverse shear stress [23] and others not listed can, however, be included in the formulation if stress or strain measurements are available for the damage detection. All these additional inclusions in the formulation will enhance the resolution of the algorithm with relevant additional measurements.

\section{The force-based approach}

\subsection{The cross-section of the element}

The cross-section of a rectangular beam element is shown in Fig. 1. Table 1 gives the parameters of the steel bars in the cross-section. Subscript $s$ on the variables denote the steel layer and the superscript $i$ denotes the $i$ th steel bar.

\subsection{Equilibrium and compatibility}

Fig. 2 shows a schematic representation of an incremental length of the beam element. The vector of forces acting on the cross-section is $D(x)=\left\{N_{B}(x) \quad M_{B}(x) \quad N_{s, b o t t o m}(x) \quad N_{s, t o p}(x)\right\}^{T}$, where $M_{B}(x)$ and $N_{B}(x)$ are respectively the bending moment and axial force acting on the beam section, $N_{s, t o p}(x)$ and $N_{s, b o t t o m}(x)$ are the axial forces carried by the equivalent steel layers and the subscripts top and bottom denote the top and bottom of the beam respectively. Subscript $s$ denotes the steel layer and $B$ denotes the beam. $D_{b}(x)=\left\{D_{b, \text { bottom }}(x) \quad D_{b, \text { top }}(x)\right\}^{T}$ is vector of interface forces, where subscript $b$ denotes 
the bonding at the interface. Since only the self-weight $p_{y}(x)$ exists in the finite element, the load vector may be written as $p(x)=\left\{\begin{array}{llll}0 & p_{y}(x) & 0 & 0\end{array}\right\}^{T}$. The equilibrium equation of the beam segment becomes

$$
\partial^{T} D(x)-\partial_{b}^{T} D_{b}(x)-p(x)=0
$$

where

$$
\partial=\left[\begin{array}{cccc}
\frac{d}{d x} & 0 & 0 & 0 \\
0 & \frac{d^{2}}{d x^{2}} & 0 & 0 \\
0 & 0 & \frac{d}{d x} & 0 \\
0 & 0 & 0 & \frac{d}{d x}
\end{array}\right] \text { and } \partial_{\mathrm{b}}=\left[\begin{array}{ccccc}
-1 & y_{\text {bottom }} \frac{d}{d x} & 1 & 0 \\
-1 & y_{\text {top }} \frac{d}{d x} & 0 & 1
\end{array}\right]
$$

The displacement vector of the section is $u(x)=\left\{u_{B}(x) v_{B}(x) u_{s, b o t t o m}(x) u_{s, t o p}(x)\right\}^{T}$, where $v_{B}(x)$ and $u_{B}(x)$ are the transverse and axial displacements of the beam section and $u_{s, t o p}(x)$ and $u_{s, b o t t o m}(x)$ denote the axial displacement of the equivalent steel layer at the top and bottom respectively of the beam. The associated vector of deformation of the beam section is $d(x)=\left\{\varepsilon_{B}(x) \kappa_{B}(x) \quad \varepsilon_{s, b o t t o m}(x) \quad \varepsilon_{\text {s,top }}(x)\right\}^{T}$. The two vectors are related as $d(x)=\partial u(x)$ with the small deformation assumption. The bond-interface slips are determined through the following compatibility relationship between the beam section and the displacement of the bar layers as

$$
\begin{gathered}
u_{b, \text { bottom }}(x)=u_{s, \text { bottom }}(x)-u_{B}(x)+y_{\text {bottom }} \frac{d v_{B}(x)}{d x} \\
u_{b, \text { top }}(x)=u_{s, \text { top }}(x)-u_{B}(x)+y_{\text {top }} \frac{d v_{B}(x)}{d x}
\end{gathered}
$$

The compatibility relation of the interface between concrete and steel layers can be written in matrix form as $d_{b}(x)=\partial_{b} u(x)$, where $d_{b}(x)=\left\{u_{b, b o t t o m}(x) u_{b, \text { top }}(x)\right\}^{T}$ is the slip vector with $u$ denotes the slip at the interface around the steel layers.

\subsection{Force-deformation relation}

The force-deformation relations of a reinforced concrete section and its bond interfaces depend on the properties of the constituent material and the cross-sectional geometry of the component. Such relations can be expressed as 


$$
\begin{aligned}
d(x) & =f_{B}(x) D(x) \\
d_{b}(x) & =f_{b}(x) D_{b}(x)
\end{aligned}
$$

in which $f_{b}(x)$ and $f_{B}(x)$ are the flexibility matrices of the bond interface and the beam section respectively. They can be written as follows based on the Euler-Bernoulli beam theory:

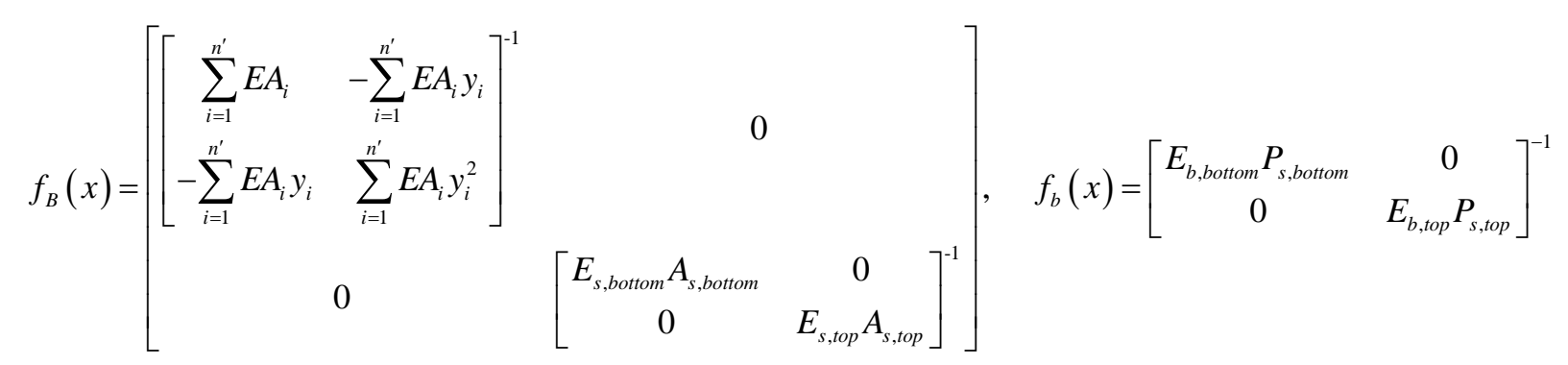

with $A_{i}$ denotes the area of the ith concrete layer; $E$ denotes the elastic modulus of the concrete $y_{i}$ denotes the distance between the ith concrete layer and the neutral axis of the beam section; $n^{\prime}$ is the number of the concrete layer; $E_{s, b o t t o m}, E_{s, \text { top }}$ and $E_{b, b o t t o m}, E_{b, \text { top }}$ denote the elastic modulus and the equivalent bond stiffness of steel respectively.

\subsection{Force-based element}

The generalized forces on the force-based layered element in local reference coordinates without rigid body displacement modes are shown in Fig. 3. The layered force-based finite element can be formulated from the principle of minimum complementary potential energy as

$$
\int_{L}[N(x)]^{T}[f(x)][N(x)]\left\{\begin{array}{c}
\bar{Q} \\
\bar{Q}_{b}
\end{array}\right\} d x=\left\{\begin{array}{c}
\bar{U} \\
0
\end{array}\right\}
$$

where $[N(x)]$ is the force shape function matrix, and

$$
N(x)=\left[\begin{array}{cc}
N_{B B}(x) & N_{B b}(x) \\
N_{b B}(x) & N_{b b}(x)
\end{array}\right]
$$

with $N_{B B}(x), N_{B b}(x), N_{b B}(x)$ and $N_{b b}(x)$ defined in the Appendix. $[f(x)]$ is the flexibility matrix of the beam, and

$$
f(x)=\left[\begin{array}{cc}
f_{B}(x) & 0 \\
0 & f_{b}(x)
\end{array}\right]
$$


and the elemental nodal force vector without considering the rigid body modes is written as

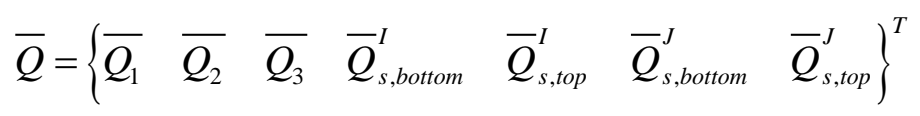

where $\overline{Q_{1}}$ is the element nodal axial force; $\overline{Q_{2}}$ and $\overline{Q_{3}}$ are the element nodal moments as shown in Fig. 3; the superscripts $I$ and $J$ denote the node numbers of the element.

$\bar{U}$ is the nodal displacement vector without considering the rigid body modes, with

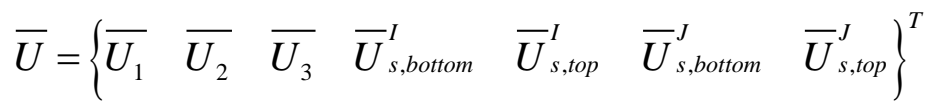

$\overline{U_{1}}, \overline{U_{2}}$ and $\overline{U_{3}}$ are the nodal displacement corresponding to $\overline{Q_{1}}, \overline{Q_{2}}$ and $\overline{Q_{3}}$.

$\bar{Q}_{b}$ is the bond interface force at $m-1$ selected reference points as defined below, and

$$
\bar{Q}_{b}=\left\{\begin{array}{llllll}
\bar{Q}_{b, \text { bottom }}^{2} & \cdots & \bar{Q}_{b, \text { bottom }}^{m} & \bar{Q}_{b, \text { top }}^{2} & \cdots & \bar{Q}_{b, \text { top }}^{m}
\end{array}\right\}^{T}
$$

In this element, the shape function for the force is the most important factor for the determination of the bond interface force distribution along the steel layers. The assumed bond interface force distribution along the interface between the beam component and the steel layers can be written in term of the bond interface forces at $m$ points using the Lagrange interpolation function as follows

$$
D_{b, \text { top }}(x)=\sum_{i=1}^{m} l_{i}(x) \bar{Q}_{b, \text { top }}^{i}, \quad D_{b, \text { bottom }}(x)=\sum_{i=1}^{m} l_{i}(x) \bar{Q}_{b, b o t t o m}^{i}
$$

where $l_{i}(x)=\frac{\left(x-x_{1}\right) \cdots\left(x-x_{i-1}\right)\left(x-x_{i+1}\right) \cdots\left(x-x_{m}\right)}{\left(x_{i}-x_{1}\right) \cdots\left(x_{i}-x_{i-1}\right)\left(x_{i}-x_{i+1}\right) \cdots\left(x_{i}-x_{m}\right)} \quad(i=1,2, \cdots, m)$

The bond-interface forces at selected reference points are not totally independent because one of them can be expressed in terms of the remaining bond-interface forces of the steel layer nodal forces after enforcing the external axial force equilibrium condition in the steel layer

$$
\int_{x}^{L} D_{b, \text { bottom }}(x) d x=\bar{Q}_{s, \text { bottom }}^{I}+\bar{Q}_{s, \text { bottom }}^{J}, \quad \int_{x}^{L} D_{b, \text { top }}(x) d x=\bar{Q}_{s, \text { top }}^{I}+\bar{Q}_{s, \text { top }}^{J}
$$

The axial force in the steel layers, the bending moment and axial force in the beam section are determined from 


$$
\begin{aligned}
& N_{s, \text { botom }}(x)=\int_{0}^{x} D_{b, \text { bottom }}(x) d x-\bar{Q}_{s, \text { botom }}^{I} \\
& N_{s, \text { top }}(x)=\left(\int_{0}^{x} D_{b, \text { top }}(x) d x-\bar{Q}_{s, \text { top }}^{I}\right) \\
& M_{B}(x)=\left(\frac{x}{L}-1\right) \bar{Q}_{2}+\frac{x}{L} \bar{Q}_{3}+\left(1-\frac{x}{L}\right)\left(y_{\text {bottom }} \bar{Q}_{s, \text { botom }}^{I}+y_{\text {top }} \bar{Q}_{s, \text { top }}^{I}\right)-\frac{x}{L}\left(y_{\text {botom }} \bar{Q}_{s, \text { botom }}^{J}+y_{\text {top }} \bar{Q}_{s, \text { top }}^{J}\right)+\left(y_{\text {bottom }} N_{s, \text { bottom }}(x)+y_{\text {top }} N_{s, \text { top }}(x)\right) \\
& N_{B}(x)=\bar{Q}_{1}+\left(\bar{Q}_{s, \text { botom }}^{I}+\bar{Q}_{s, \text { top }}^{I}-\int_{0}^{x} D_{b, \text { botom }}(x) d x-\int_{0}^{x} D_{b, \text { top }}(x) d x\right)
\end{aligned}
$$

From Eqs. (10) to (12), all of the force shape functions can be determined [14] and they are listed in the Appendix of this paper.

Then Eq. (4) can be written as

$$
\left[\begin{array}{cc}
F_{B B}(x) & F_{B b}(x) \\
F_{b B}(x) & F_{b b}(x)
\end{array}\right]\left\{\begin{array}{c}
\bar{Q} \\
\bar{Q}_{b}
\end{array}\right\}=\left\{\begin{array}{c}
\bar{U} \\
0
\end{array}\right\}
$$

where

$$
\begin{aligned}
& F_{B B}=\int_{0}^{L} N_{B B}^{T}(x) f_{B}(x) N_{B B}(x) d x+\int_{0}^{L} N_{b B}^{T}(x) f_{b}(x) N_{b B}(x) d x \\
& F_{B b}=\int_{0}^{L} N_{B B}^{T}(x) f_{B}(x) N_{B b}(x) d x+\int_{0}^{L} N_{b B}^{T}(x) f_{b}(x) N_{b b}(x) d x=F_{b B}^{T} \\
& F_{b b}=\int_{0}^{L} N_{B b}^{T}(x) f_{B}(x) N_{B b}(x) d x+\int_{0}^{L} N_{b b}^{T}(x) f_{b}(x) N_{b b}(x) d x
\end{aligned}
$$

The element flexibility matrix without considering the rigid body modes can be obtained from Eqs. (12) and (13) as

$$
\bar{F}^{e}=F_{B B}-F_{B b} F_{b b}^{-1} F_{b B}
$$

and the elemental stiffness matrix without considering the rigid body modes can be obtained as $\bar{K}^{e}=\left[\bar{F}^{e}\right]^{-1}$

The above relation between element stiffness matrix with and without considering the rigid body modes is derived with the assumption of no energy loss in the element modes. When the rigid body modes are included, this relation can be written as

$$
K^{e}=T_{R B M}^{T} \bar{K}^{e} T_{R B M}
$$


In this paper, four points along the element are taken to construct the Lagrange interpolation function, and matrix $T_{R B M}$ is listed in the Appendix.

\section{Damage identification}

The steel bars are assumed to have deficiency in bonding with the surrounding concrete. The equivalent steel layers are therefore intact while the bonding interface layers and the concrete are damaged.

The stiffness matrix of the beam with damage can be assembled from the elemental stiffness matrix $K^{e}$ as

$$
K=\sum_{i=1}^{n} T_{i}^{T} T_{R B M}^{T} \bar{K}_{i}^{e} T_{R B M} T_{i}
$$

where $T_{i}$ is the transformation matrix.

The force-displacement of the damaged structure are related by

$$
F=(K-\Delta K)(U-\Delta U)
$$

where $F$ is the force vector. $\Delta K$ is the stiffness reduction matrix of the system due to the damage. $K$ and $U$ are the stiffness matrix and nodal deformation vector of the intact beam. The latter can be computed from $U=K^{-1} F$ under the static load.

The vector of displacement differences $\Delta U$ obtained from the structures with and without damage can be obtained from Eq. (18) as

$$
\Delta U=K^{-1} \Delta K K^{-1} F+K^{-1} \Delta K \Delta U \approx K^{-1} \Delta K K^{-1} F
$$

For the de-bonding in steel bar, the associated stiffness changes $\Delta K$ can be expressed as

$$
\Delta K=\sum_{i=1}^{n} T_{i}^{T}\left(\frac{\partial K_{i}^{e}}{\partial E} \Delta E+\frac{\partial K_{i}^{e}}{\partial E_{b, \text { bottom }}} \Delta E_{b, \text { bottom }}+\frac{\partial K_{i}^{e}}{\partial E_{b, \text { top }}} \Delta E_{b, \text { top }}\right) T_{i}
$$

where $n$ is the number of finite element, and $K_{i}^{e}$ is the ith elemental stiffness matrix; increments $\Delta E_{b, b o t t o m}, \Delta E_{b, t o p}$ and $\Delta E$ denote respectively the change of the bond stiffness and the elastic modulus of concrete obtained from the intact and damaged states of the beam. 


$$
\begin{gathered}
\frac{\partial K_{i}^{e}}{\partial E}=-T_{R B M}^{T} \bar{K}_{i}^{e} \frac{\partial \bar{F}_{i}^{e}}{\partial E} \bar{K}_{i}^{e} T_{R B M} \\
\frac{\partial K_{i}^{e}}{\partial E_{b, b o t o m}}=-T_{R B M}^{T} \bar{K}_{i}^{e} \frac{\partial \bar{F}_{i}^{e}}{\partial E_{b, b o t o m}} \bar{K}_{i}^{e} T_{R B M}, \quad \frac{\partial K_{i}^{e}}{\partial E_{b, \text { top }}}=-T_{R B M}^{T} \bar{K}_{i}^{e} \frac{\partial \bar{F}_{i}^{e}}{\partial E_{b, \text { top }}} \bar{K}_{i}^{e} T_{R B M}
\end{gathered}
$$

where

$$
\begin{aligned}
& \frac{\partial \bar{F}_{i}^{e}}{\partial E}=\frac{\partial F_{B B}}{\partial E}-\frac{\partial F_{B b}}{\partial E}\left(F_{b b}^{-1} F_{b B}\right)-F_{B b}\left(F_{b b}^{-1} \frac{\partial F_{b B}}{\partial E}-F_{b b}^{-1} \frac{\partial F_{b b}}{\partial E} F_{b b}^{-1} F_{b B}\right) \\
& \frac{\partial \bar{F}_{i}^{e}}{\partial E_{b, \text { bottom }}}=\frac{\partial F_{B B}}{\partial E_{b, b o t t o m}}-\frac{\partial F_{B b}}{\partial E_{b, b o t t o m}}\left(F_{b b}^{-1} F_{b B}\right)-F_{B b}\left(F_{b b}^{-1} \frac{\partial F_{b B}}{\partial E_{b, \text { bottom }}}-F_{b b}^{-1} \frac{\partial F_{b b}}{\partial E_{b, b o t o m}} F_{b b}^{-1} F_{b B}\right) \\
& \frac{\partial \bar{F}_{i}^{e}}{\partial E_{b, \text { top }}}=\frac{\partial F_{B B}}{\partial E_{b, \text { top }}}-\frac{\partial F_{B b}}{\partial E_{b, \text { top }}}\left(F_{b b}^{-1} F_{b B}\right)-F_{B b}\left(F_{b b}^{-1} \frac{\partial F_{b B}}{\partial E_{b, t o p}}-F_{b b}^{-1} \frac{\partial F_{b b}}{\partial E_{b, t o p}} F_{b b}^{-1} F_{b B}\right)
\end{aligned}
$$

The identification is conducted with a model of the local elemental damage whereby the damage from cracking and local bond damage are separated modelled without considering the coupling effect. The problem then becomes a parameter identification problem. Two types of damage indicators, $\boldsymbol{\alpha}_{\mathbf{B}}, \boldsymbol{\alpha}_{\mathbf{b}}$, are defined to describe those two types of damage. Success identification of these damage indicators would mean successful identification of the two types of damages.

Eqs. (19) and (20) can be combined to give

$$
\begin{aligned}
\Delta U & =K^{-1} \sum_{i=1}^{n} T_{i}^{T}\left(\alpha_{B i} \Delta K_{B i}^{e}+\alpha_{b i} \Delta K_{b i}^{e}\right) T_{\mathrm{i}} K^{-1} F \\
& =\sum_{i=1}^{n} \alpha_{B i} \vec{K}_{B i}+\sum_{i=1}^{n} \alpha_{b i} \vec{K}_{b i}
\end{aligned}
$$

where

$$
\begin{aligned}
& \vec{K}_{B i}=K^{-1} \sum_{i=1}^{n} T_{i}^{T} \Delta K_{B i}^{e} T_{\mathrm{i}} K^{-1} F, \quad \vec{K}_{b i}=K^{-1} \sum_{i=1}^{n} T_{i}^{T} \Delta K_{b i}^{e} T_{\mathrm{i}} K^{-1} F
\end{aligned}
$$

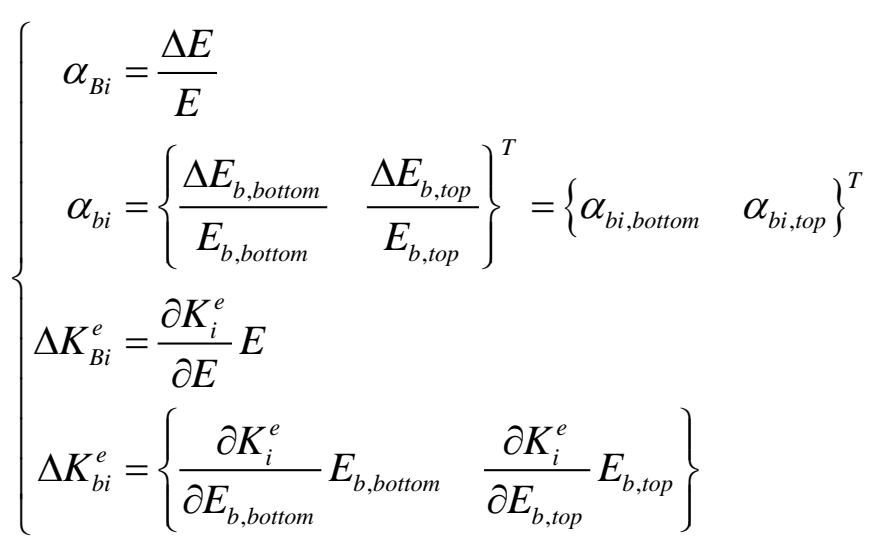


The practical scenario with limited displacements is studied. Let the Boolean matrix, Q, relates the vector of measured response to all the degrees-of-freedom of the system. The error vector between the measured and predicted displacement differences is then expressed as:

$$
e\left(\alpha_{B}, \alpha_{b}\right)=Q \Delta U-\Delta U_{s}
$$

where $\Delta U_{s}$ is the vector of measured displacement differences obtained from the two different states of the structures.

\section{Damage identification algorithm}

\subsection{When only one type of damage exists in the structure}

If only one type of damage exist in the structure, Eq. (25) can be expressed as.

$$
e(\alpha)=Q \Delta U-\Delta U_{s}
$$

where $\alpha$ represents one of $\alpha_{B}$ and $\alpha_{b} ; \Delta U$ and $\Delta U_{s}$ represent, respectively, the analytical and the measured displacement difference associated with $\alpha$.

The solution of the damage identification problem is via the minimization of a least-squares error function with the quadratic programming technique [22], and the error function is written as

$$
J(\alpha)=\frac{1}{2} \alpha^{T} A \alpha+C^{T} \alpha+\frac{1}{2} \Delta U_{s}^{T} \Delta U_{s}
$$

with $A=\left(Q K^{-1} \Delta K K^{-1} F\right)^{T}\left(Q K^{-1} \Delta K K^{-1} F\right) ; C=-\Delta U_{s}^{T} Q K^{-1} \Delta K K^{-1} F$; and $0 \leq \alpha \leq 1$. The algorithm is iterative as shown below.

1) Initially assume that $\alpha_{0}=\{0,0, \cdots, 0\}^{T}$;

2) Identify damage index $\alpha_{j}$ with $j=1$ by minimizing $J^{\prime}(\alpha)=\frac{1}{2} \alpha^{T} A \alpha+C^{T} \alpha$

3) Check the convergence of computation with the following two errors as

$$
\left\{\begin{array}{l}
\text { Error } 1=J^{\prime}(\alpha)+\frac{1}{2} \Delta U_{s}^{T} \Delta U_{s} \\
\text { Error } 2=\frac{\left\|\alpha_{j+1}-\alpha_{j}\right\|}{\left\|\alpha_{j}\right\|} \times 100 \%
\end{array}\right.
$$


where $\alpha_{j}, \alpha_{j+1}$ are the indices obtained in the $j^{\text {th }}$ and $j+1^{\text {th }}$ iterations. Solution is considered converged when both the above errors are smaller than the pre-defined threshold of $1 \times 10^{-20}$ and $1 \times 10^{-6}$ respectively.

4) If the computation does not converge, let $\alpha_{0}=\alpha_{j+1}$ and repeat Steps 1 to 3 until convergence is achieved.

\subsection{When both types of damages exist in the structure}

If both types of damages exist in the structure, Eq. (25) becomes

$$
e\left(\alpha_{B}, \alpha_{b}\right)=Q\left[\sum_{i=1}^{n} \vec{K}_{B i} \quad \sum_{i=1}^{n} \vec{K}_{b i}\right]\left\{\begin{array}{l}
\alpha_{B} \\
\alpha_{b}
\end{array}\right\}-\Delta U_{s}
$$

Since the bonding stiffness is very small compared with that from the beam itself, the bonding damage would be difficult to detect using the above method when both types of damages exist in the beam element. In this study, the Newton iteration method is applied to solve this problem, and the increment of the bonding damage index $\Delta \alpha_{b}$ will be normalized with multiplier $\beta$ as

$$
\beta=\frac{\left\|Q \sum_{i=1}^{n} \vec{K}_{B i}\right\|}{\left\|Q \sum_{i=1}^{n} \vec{K}_{b i}\right\|}
$$

\section{Verification with numerical simulations}

The numerical simulations are based on data generated from a $4 \mathrm{~m}$ long rectangular uniform simply supported reinforced concrete beam with $300 \mathrm{~mm}$ high and $200 \mathrm{~mm}$ wide cross-section. Three 16-mm diameter mild steel bars and two 6-mm-diameter steel bars are located respectively at the bottom and top of the beam section. Shear reinforcements are in the form of 6-mm diameter links at $195 \mathrm{~mm}$ spacing. The compression strength, tensile strength, Poisson ratio, modulus of elasticity and density of concrete and bonding stiffness between steel and concrete are respectively 54.4 MPa, 3.77 MPa, 0.16, $30.2 \mathrm{GPa}, 2351.4 \mathrm{~kg} / \mathrm{m}^{3}$ and $9.05 \mathrm{MPa} / \mathrm{mm}$. The yield stress and elastic modulus of the steel bars are $300.07 \mathrm{MPa}$ and $181.53 \mathrm{GPa}$ respectively. There are twelve equal layered force-based finite elements in the beam model.

A single $5.0 \mathrm{kN}$ static force is applied at one third, half and two-third span of the beam in turn to check on the effect of different loading locations. Displacements at 5 and 11 locations evenly 
distributed along the beam are used for the identification. These loading and sensor arrangements are adopted for the following studies unless otherwise stated.

Three different bonding loss scenarios are studied: (a) 10\% reduction in element 4; (b) 15\% reduction in element 4 and 10\% reduction in elements 3 and 5 to simulate bonding loss in a smeared zone; and (c) 10\% reduction in elements 4 and 9. The identification problem is conducted according to the procedure described above.

\subsection{Identification of local bonding loss}

Displacements from five locations are used for the identification. Figures 4 to 6 give the identified damage index, $\alpha_{b}$, alongside the true values for the three bonding loss scenarios with different loading positions. The damage indices match the true values very well and the loading position is found not significant to the accuracy of identification.

Although the contribution of the bonding stiffness to the overall stiffness of the beam element is small, yet the single and multiple bonding damages can be estimated accurately with the proposed refined model. The bond interface distribution in a smeared zone can also be estimated satisfactory as shown. It may be concluded that the proposed strategy is accurate and effective to determine the bonding damage location and extent with different loading position.

\subsection{Identification of local bonding loss with noisy measurements}

The parameters for this study are the same as those used above with the static load applied at one-third length of the beam. $1 \%, 5 \%$ and $10 \%$ normal random noise are included in the measurements. The identified results for the different bond loss scenarios are shown in Figures 7 to 9. The identified bonding damage indices, $\alpha_{b}$, is found not sensitive to the noise level when single bond loss is involved. The noise effect does affect the identified results when the de-bonding occurs in a zone over several beam element as shown in Figure 8. However, the set of results can be improved significantly when the number of measured data is increased to eleven. The measurement noise also has some effect on the identified results in the multiple loss scenario.

\subsection{Identification of local beam damage and bonding loss in a single element}

The parameters for this study are the same as those used above with the static load applied at one-third length of the beam. The same noise levels as for last study are considered, and the static displacements measured at eleven locations are used for the identification. Figures 10 to 12 show the identified results for the different damage scenarios. The identified damage indices are found close to the true values while the identified results for the bonding loss is slightly poorer than those 
for the beam damage. This is due to the small contribution of the bonding stiffness to the stiffness of the beam element, and the normalization of the damage index in Eq. (27) is useful in improving the effectiveness of the proposed strategy to identify the de-bonding loss. It may be concluded that the proposed method is effective to estimate the damage location and extent even with the existence of both the local bonding loss and local beam damage.

\subsection{Identification of beam and bonding damage with finer finite element mesh size}

In order to study the effect of the finite element mesh size on the identified results, the same parameters of local beam damage and bonding loss are adopted, but the element size is reduced to $50 \%$ of the original, which means there are 24 beam elements in total and the number of candidate damaged elements is doubled in this case. The noise levels considered in previous studies are adopted, and static measurements from eleven points evenly distributed along the beam are used for the identification. The identified results are shown in Figs. 13 to 15 for the same three damage scenarios studied earlier. The identified results are close to the true values with acceptable accuracy. Compared with results in Figs. 10 to 12, the error of identification is larger when there is the same number of measurements. This shows that a finer finite element mesh would give less accurate results compared to those from a rougher finite element mesh as shown in Section 5.3, especially for the case with beam and bonding damage in multiple elements in a group. Figs. 16 to 18 show the identified results from 23 displacement measurements along the beam. The identified results have similar accuracy with the results in Figs. 10 to 12. This show that more sensors are needed with a finer mesh model to give similar identification accuracy.

There is also a dilemma in the solution of the algorithm with a more complex model of the damages. The computation technique generally adopted is based on singular value decomposition or regularization to improve the estimate of the solution whereas the accuracy depends on the condition of the measurement matrix. There is no guarantee that more measurements would lead to better estimates. This is also noted in this section that a finer finite element mesh does not necessarily yield better results. The resolution of the solution technique is not capable to differentiate small differences in different types of damages, and only an 'equivalent' damage is given representing the anomaly modelled and other side effects that have not been modelled in the algorithm.

\section{Conclusions}


A damage identification strategy on the occurrence of de-bonding of steel bars in concrete is formulated in this paper with the development of a layered force-based finite beam element to refine and describe the behavior of de-bonding loss under static load. Static measured displacements are used for the identification. An error function on the displacements is least-squares minimized. Numerical simulations show that the de-bonding of the steel bar as well as local damage in the beam can be estimated effectively with the proposed method. Bonding damage in the form of a smeared zone is noted more difficult to estimate accurately than the local de-bonding at single or multiple locations, and more measured data are required for better estimation. The proposed method of normalization on the damage index when two parameters of highly different sensitivity are identified together is shown to be effective for the present study. The proposed refined layered finite element model for beam structure may be more suitable for the evaluation of the bond interface conditions in reinforced concrete. Further experimental verification is needed for its application in practice. 


\section{Appendix:}

$$
\begin{aligned}
& N_{B B}(x)=\left[\begin{array}{ccccccc}
1 & 0 & 0 & 1-g(x) & 1-g(x) & 1-g(x) & 1-g(x) \\
0 & \frac{x}{L}-1 & \frac{x}{L} & y_{\text {bottom }}\left(g(x)-\frac{x}{L}\right) & y_{\text {top }}\left(g(x)-\frac{x}{L}\right) & y_{\text {bottom }}\left(g(x)-\frac{x}{L}\right) & y_{\text {top }}\left(g(x)-\frac{x}{L}\right) \\
0 & 0 & 0 & g(x)-1 & 0 & g(x) & 0 \\
0 & 0 & 0 & 0 & g(x)-1 & 0 & g(x)
\end{array}\right] \text { (A1 } \\
& N_{B b}(x)=\left[\begin{array}{cccccc}
h 1(x) & h 2(x) & h 3(x) & h 1(x) & h 2(x) & h 3(x) \\
-y_{\text {bottom }} h 1(x) & -y_{\text {botom }} h 2(x) & -y_{\text {bottom }} h 3(x) & -y_{\text {top }} h 1(x) & -y_{\text {top }} h 2(x) & -y_{\text {top }} h 3(x) \\
-h 1(x) & -h 2(x) & -h 3(x) & 0 & 0 & 0 \\
0 & 0 & 0 & -h 1(x) & -h 2(x) & -h 3(x)
\end{array}\right] \\
& N_{b B}(x)=\left[\begin{array}{ccccccc}
0 & 0 & 0 & a(x) & 0 & a(x) & 0 \\
0 & 0 & 0 & 0 & a(x) & 0 & a(x)
\end{array}\right] \\
& N_{b b}(x)=\left[\begin{array}{cccccc}
b 1(x) & b 2(x) & b 3(x) & 0 & 0 & 0 \\
0 & 0 & 0 & b 1(x) & b 2(x) & b 3(x)
\end{array}\right]
\end{aligned}
$$

where

$$
\left\{\begin{array}{l}
g(x)=\frac{4 x^{3}}{L^{2}}-\frac{12 x^{3}}{L^{3}}+\frac{9 x^{4}}{L^{4}} \\
h 1(x)=-x+\frac{13 x^{2}}{4 L}-\frac{9 x^{3}}{2 L^{2}}+\frac{9 x^{4}}{4 L^{3}} \\
h 2(x)=-\frac{3 x^{2}}{L}+\frac{3 x^{3}}{L^{2}} \\
h 3(x)=\frac{15 x^{2}}{4 L}-\frac{21 x^{3}}{2 L^{2}}+\frac{27 x^{4}}{4 L^{3}}
\end{array}\right.
$$




\section{REFERENCES}

[1] Zimmerman D.C. and Smith S.W. Model refinement and damage location for intelligent structures. In: Tzou HS, Anderson GL, editors. Intelligent structural systems. The Netherlands: Kluwer, 1992:403-52.

[2] Doebling S.W., Farrar C.R. and Prime M.B. A summary review of vibration based damage identification methods. Shock and Vibration Digest, 1998, 30(2): 91-105.

[3] Salawu O.S. Detection of structural damage through change in frequency: a review. Engineering Structures, 1997, 19(9): 718-23.

[4] Banan M.R. and Hjelmstad K.D. Parameter estimation of structures from static response. I. Computational aspects. Journal of Structural Engineering ASCE, 1994, 120(11): 3243-58.

[5] Banan M.R. and Hjelmstad K.D. Parameter estimation of structures from static response. II. Numerical simulation studies. Journal of Structural Engineering ASCE, 1994, 120(11): 3259-83.

[6] Liu P.L. and Chian C.C. Parametric identification of truss structures using static strains. Journal of Structural Engineering ASCE, 1997, 123(7): 927-33.

[7] Wang X., Hu N., Fukunaga H. and Yao Z.H. Structural damage identification using static test data and changes in frequencies. Engineering Structures, 2001, 23: 610-21.

[8] Di Paola M. and Bilello C. An integral equation for damage identification of Euler-Bernoulli beams under static loads. Journal of Engineering Mechanics ASCE, 2004, 130(2): 225-34.

[9] Zhu X.Q. and Law S.S. A concrete-steel interface element for damage detection of reinforced concrete structures. Engineering Structures, 2007, 29: 3515-3524.

[10] Zhu X.Q., Law S.S. and Hao H. Damage assessment of reinforced concrete beams including the load environment. Structural Engineering and Mechanics, 2009, 33(6):765-779.

[11] Berto L., Simioni P. and Seatta A. Numerical modeling of bond behavior in RC structures affected by reinforced corrosion. Engineering Structures, 2008, 30(5): 1375-1385.

[12] Jendele L. and Cervenka J. Finite element modeling of reinforcement with bond. Computers \& Structures, 2006, 84: 1780-1791.

[13] Haskett M., Oehlers F.J. and Ali M.S.M. Local and global bond characteristics of steel reinforcing bars. Engineering Structures, 2008, 30: 376-383.

[14] Lee Y.-H., Joo Y.T., Lee T. and H D.-H. Mechanical properties of constitutive parameters in steel-concrete interface. Engineering Structures, 2011, 33(4): 1277-1290.

[15] Henderson I.E.J., Zhu X.Q., Uy B. and Mirza O. Dynamic behaviour of steel-concrete composite beams with different types of shear connectors. Part II: Modelling and comparison. Engineering Structures, 2015, 103: 308-317. 
[16] Song G., Du H., Mo Y.L., Hsu T.T.C. and Dhonde H. Concrete structural health monitoring using embedded piezoceramic transducers. Smart Materials and Structures, 2007, 16: 959-968.

[17] Xu B., Zhang T., Song G. and Gu H.C. Active interface debonding detection of a concretefilled steel tube with piezoelectric technologies using wavelet packet analysis. Mechanical Systems and Signal Processing, 2013, 36(1): 7-17.

[18] Zhu X.Q., Hao H. and Fan K.Q. Detection of delamination between steel bars and concrete using embedded piezoelectric actuators/sensors. Journal of Civil Structural Health Monitoring, 2013, 3(2): 105-115.

[19] Biswal S. and Ramaswamy A. Damage identification in concrete structures with uncertain but bounded measurements. Structural Health Monitoring, 2016, DOI: 10.1177/1475921716676993.

[20] Salari M.R. and Spacone E. Analysis of steel-concrete composite frames with bond-slip. Journal of Structural Engineering, ASCE, 2001, 127(11):1234-1250.

[21] Salari M. .R. and Spacone E. Finite element formulation of one-dimensional elements with bond-slip. Engineering Structures, 2001, 23(7): 815-826.

[22] Goldfarb D. and Idnani A. A numerically stable dual method for solving strictly convex quadratic problems. Mathematical Programming, 1983, 27: 1-33.

[23] Ramaswamy A., Barzegar F. and Voyiadjis G.Z. Study of layering procedures in finite element analysis of RC flexural and torsional elements. Journal of Structural Engineering ASCE, 121(12): 1773-1783. 
Table 1 - Characteristics of the steel bars

\begin{tabular}{lcc}
\hline & Bottom & Top \\
\hline $\begin{array}{l}\text { Areas of the equivalent steel } \\
\text { layers }\end{array}$ & $A_{s, \text { bottom }}=\sum A_{s, b o t t o m}^{i}$ & $A_{s, \text { top }}=\sum A_{s, \text { top }}^{i}$ \\
\hline Perimeters of the equivalent & $P_{s, \text { bottom }}=\sum P_{s, \text { bottom }}^{i}$ & $P_{s, \text { top }}=\sum P_{s, \text { top }}^{i}$ \\
steel layers & $y_{\text {bottom }}=\frac{\sum A_{s, \text { botom }}^{i} y_{s, \text { botom }}^{i}}{\sum A_{s, b o t o m}^{i}}$ & $y_{\text {top }}=\frac{\sum A_{s, \text { top }}^{i} y_{s, \text { top }}^{i}}{\sum A_{s, \text { top }}^{i}}$ \\
\hline $\begin{array}{l}\text { Distances between the neutral } \\
\text { axis and the equivalent steel }\end{array}$ & \\
layers
\end{tabular}




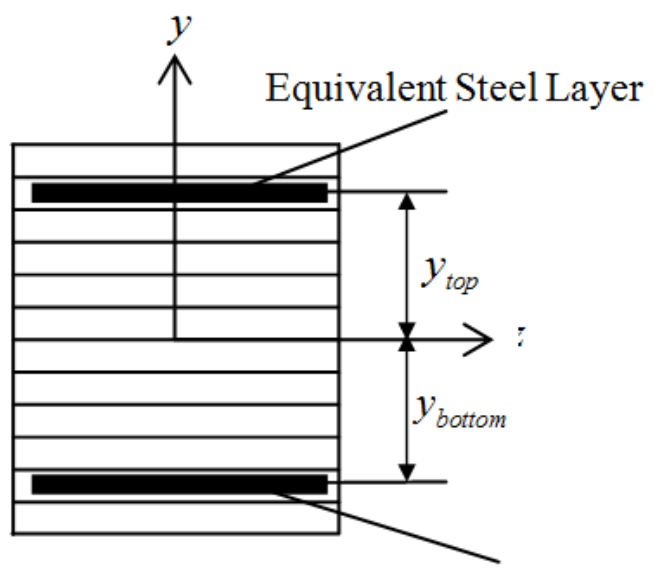

Equivalent Steel Layer

Fig. 1. Sectional view of the layered beam element

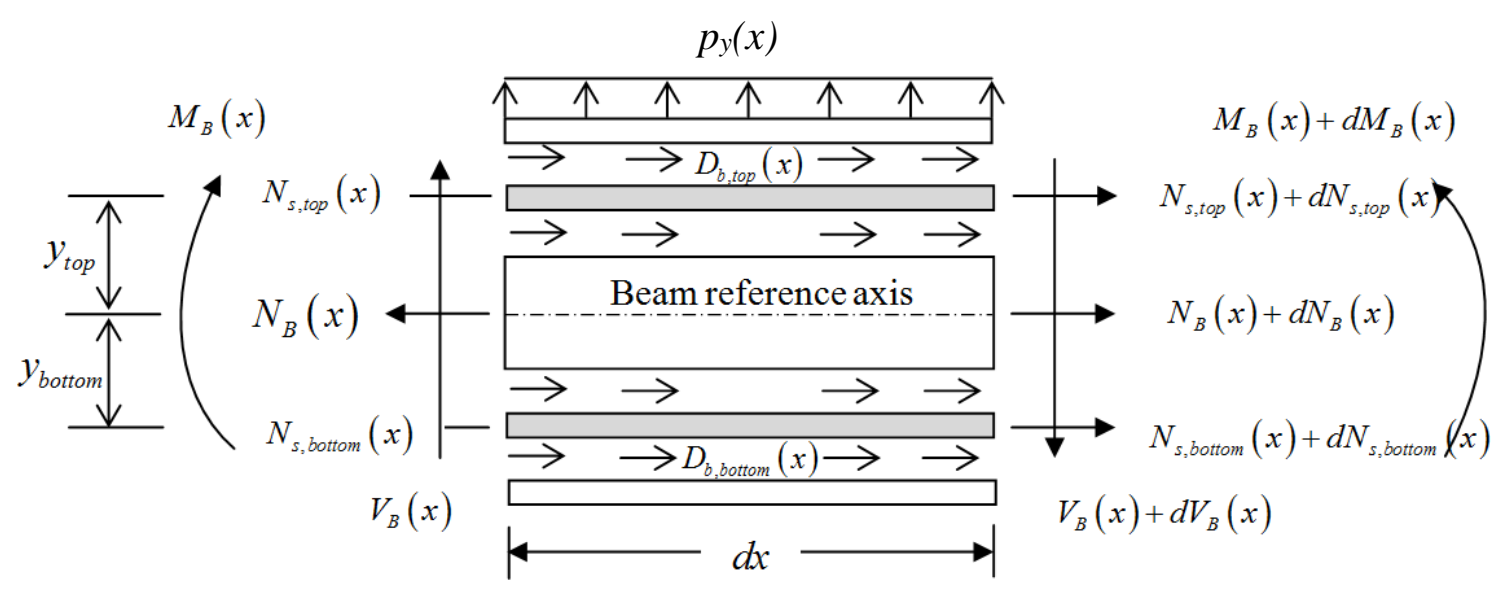

Fig. 2. Incremental length of the element with bonding
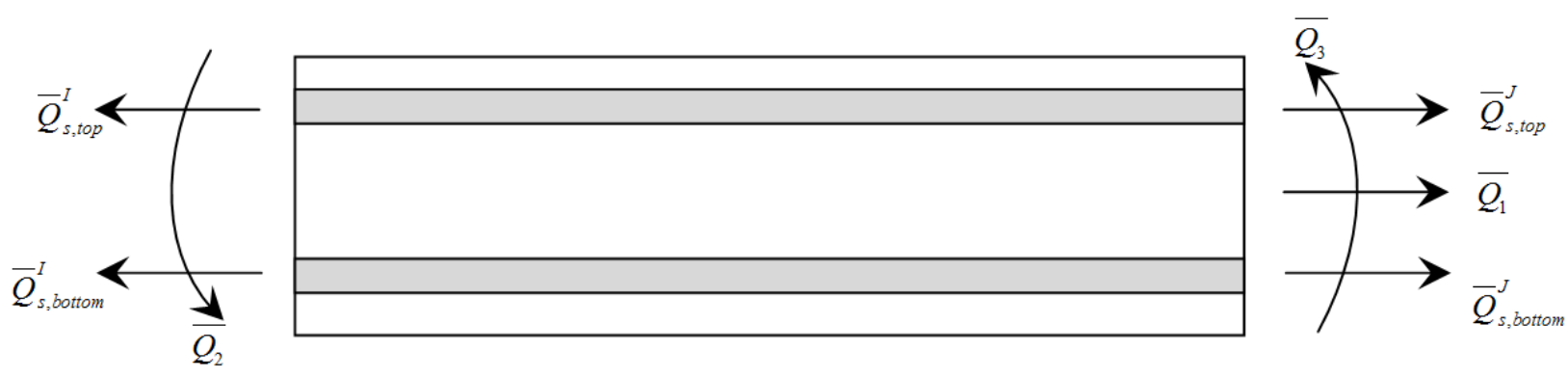

Fig. 3. Generalized forces at the beam column in local reference coordinates without considering the rigid body displacement modes 


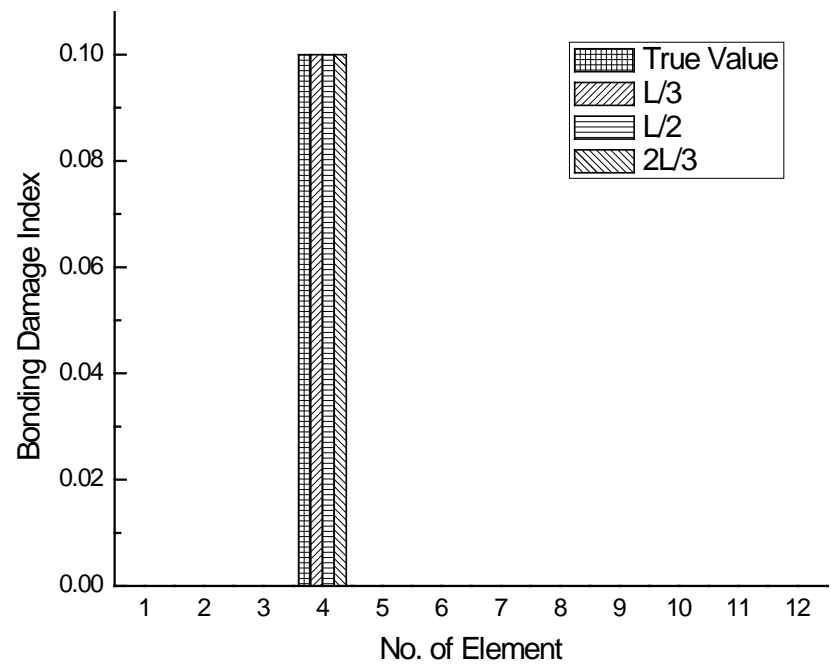

Fig. 4. Identified single bonding damage from different load locations

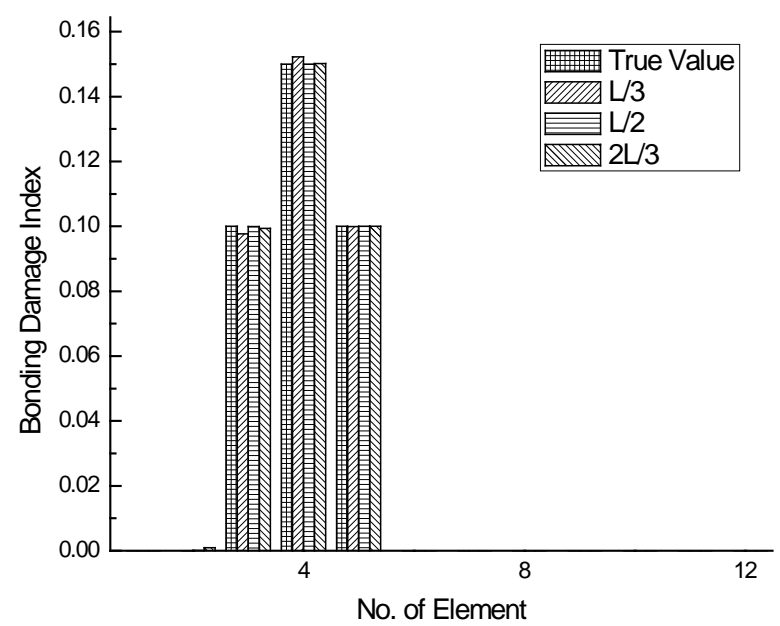

Fig. 5. Identified multiple bonding damage in a zone from different load locations 


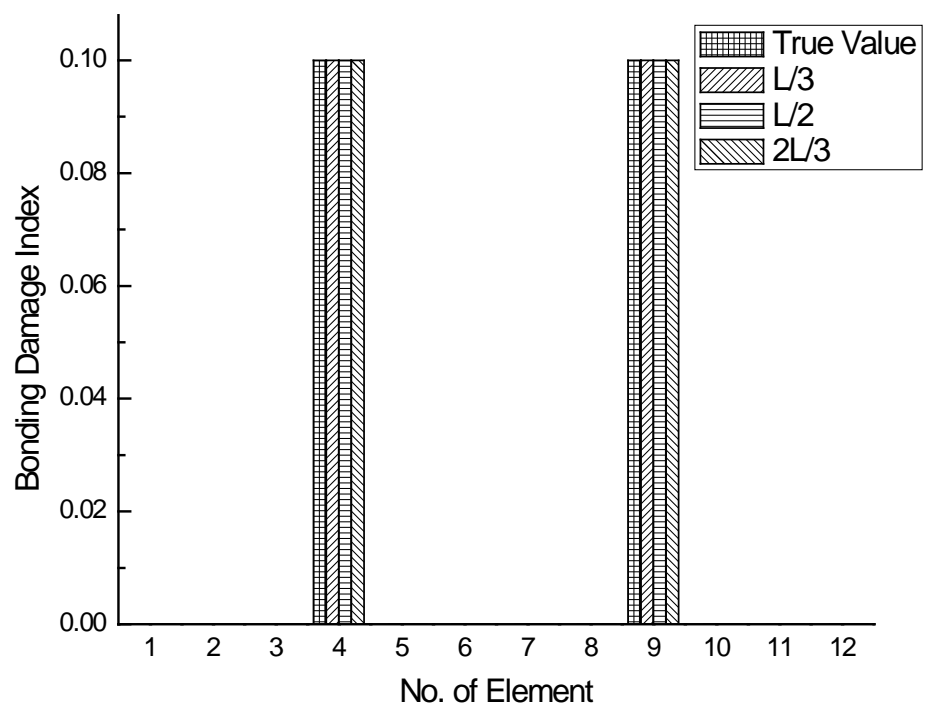

Fig. 6. Identified local bonding damage in separate element from different load locations

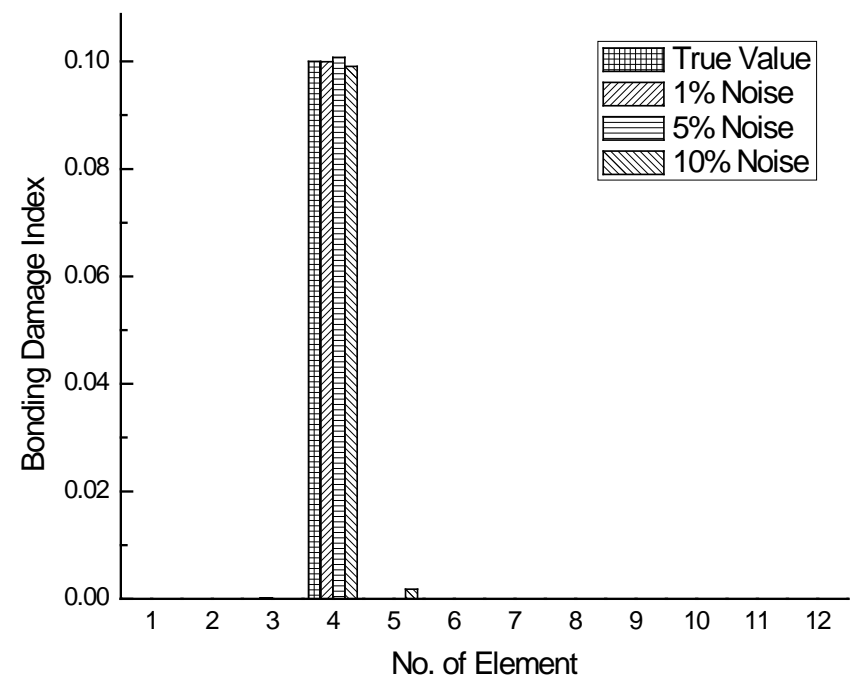

Fig. 7. Identified single bonding damage with different noise level 


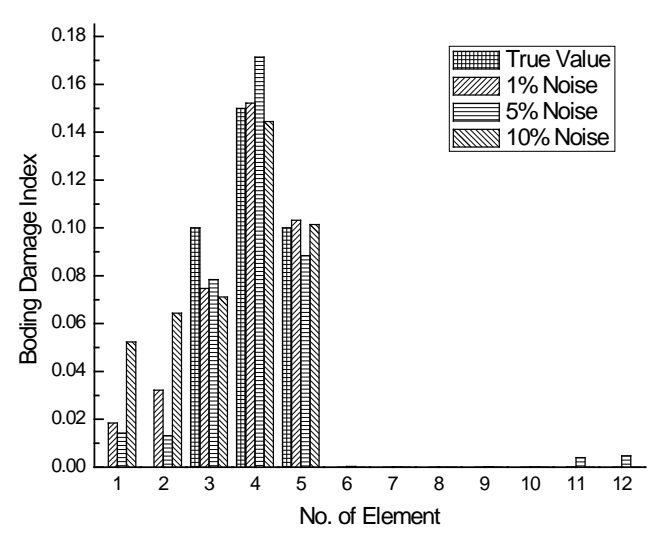

a) Five sensors

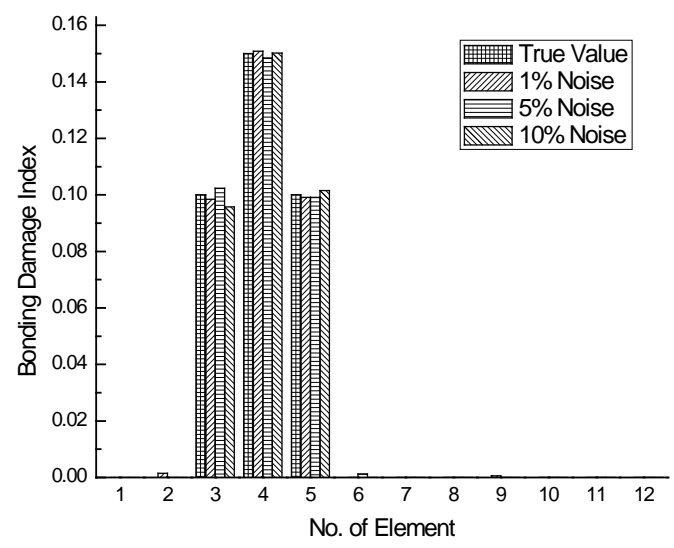

b) Eleven sensors

Fig. 8. Identified bonding damage in a zone with different noise level

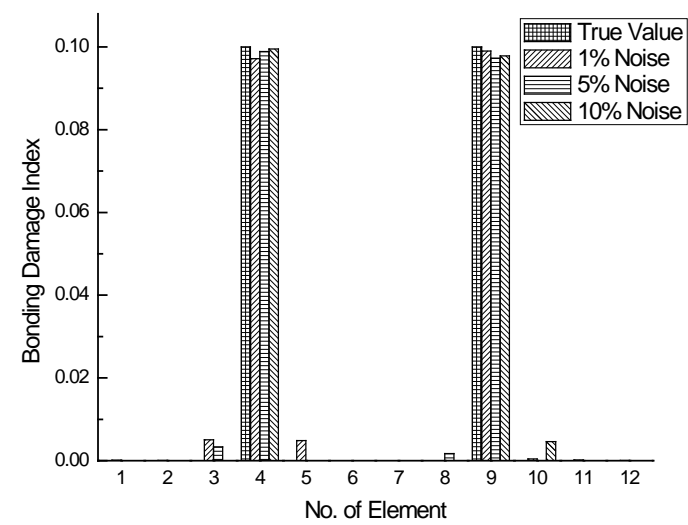

c) Five sensors

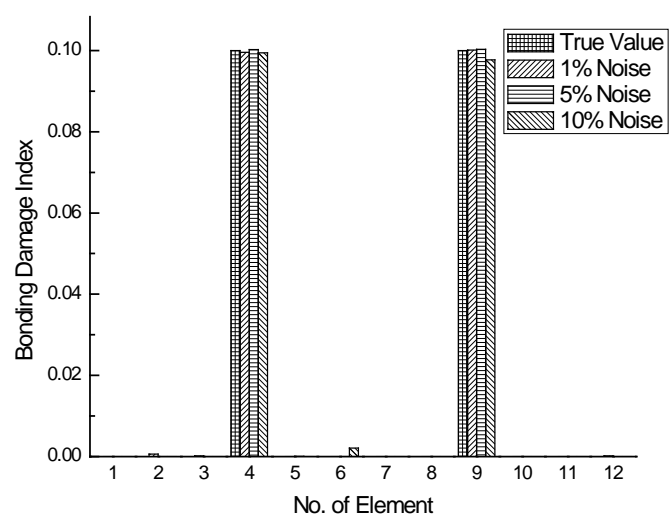

d) Eleven sensors

Fig. 9. Identified local bonding damage in separate elements with different noise level 


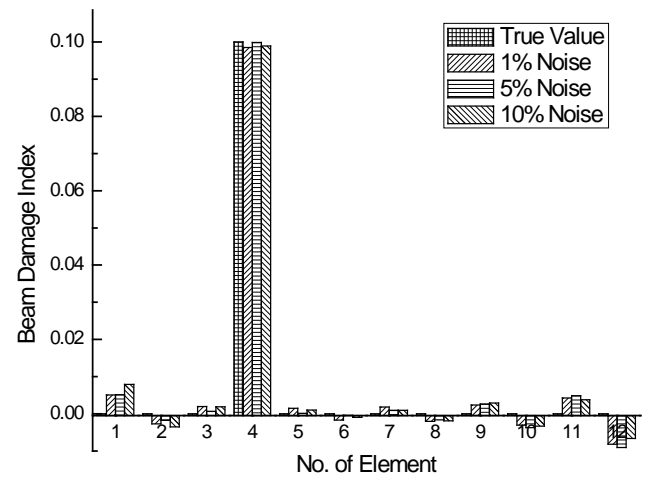

a) Beam damage index

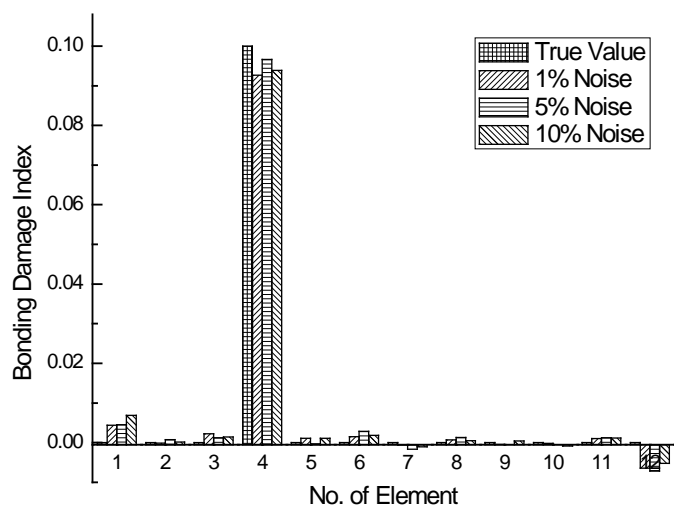

b) Bonding damage index

Fig. 10. Identified beam and bonding damage in a single element

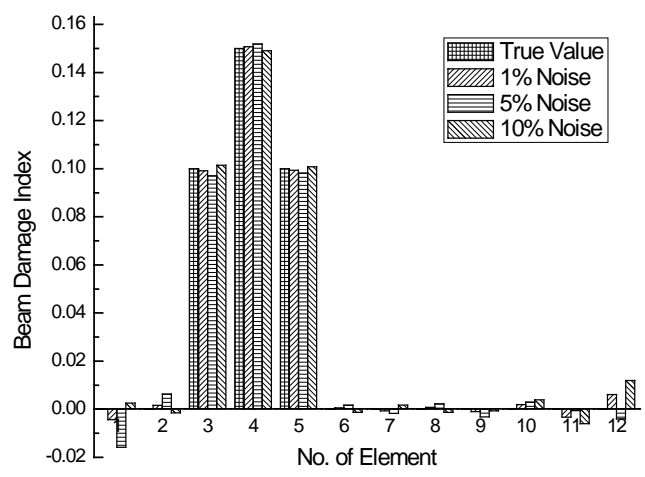

a) Beam damage index

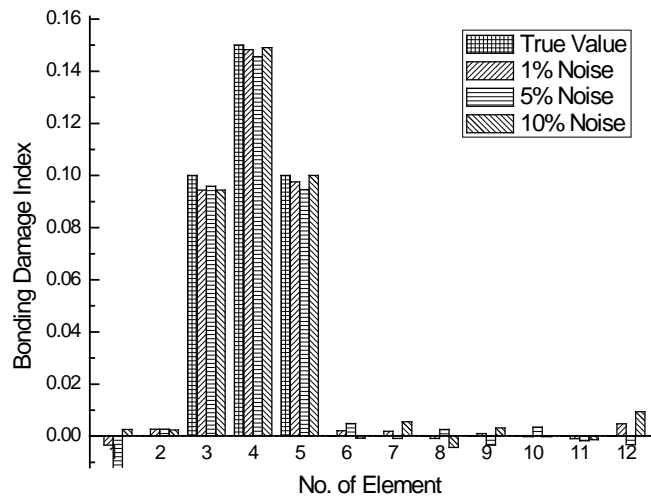

b) Bonding damage index

Fig. 11. Identified beam and bonding damage in multiple elements in a group 


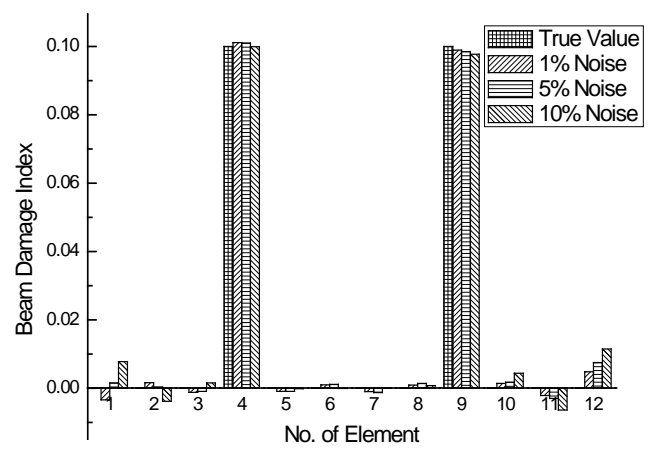

a) Beam damage index

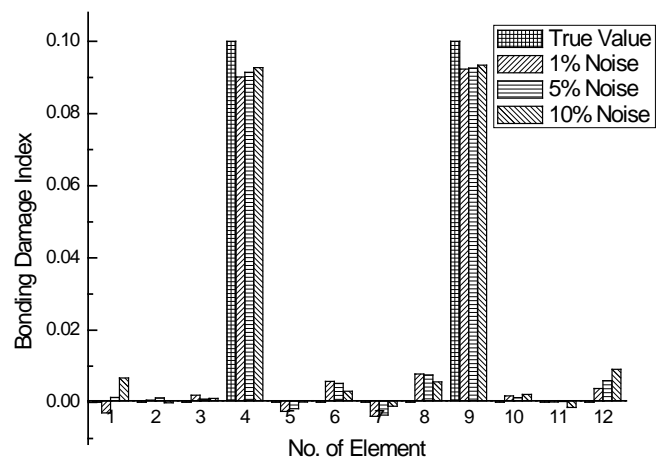

b) Bonding damage index

Fig. 12. Identified beam and bonding damage in two separate elements

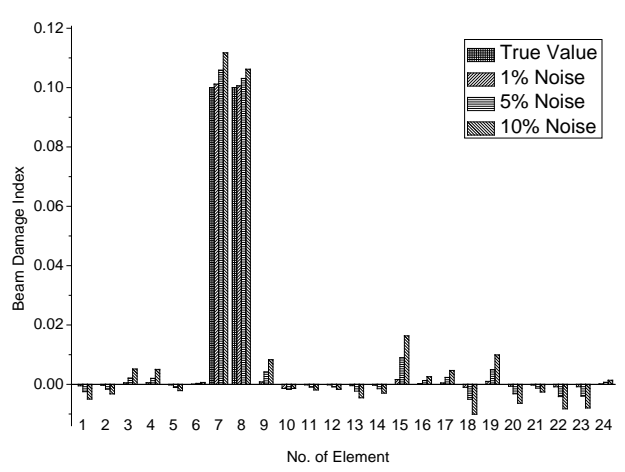

a) Beam damage index

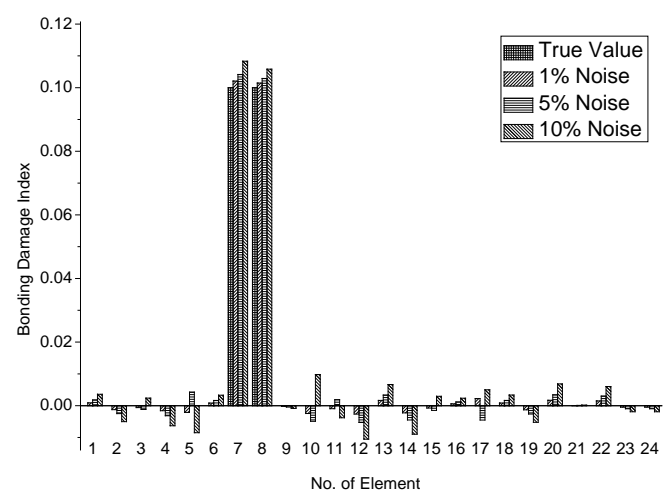

b) Bonding damage index

Figure 13 Identified beam and bonding damage in a single element with 11 sensors 


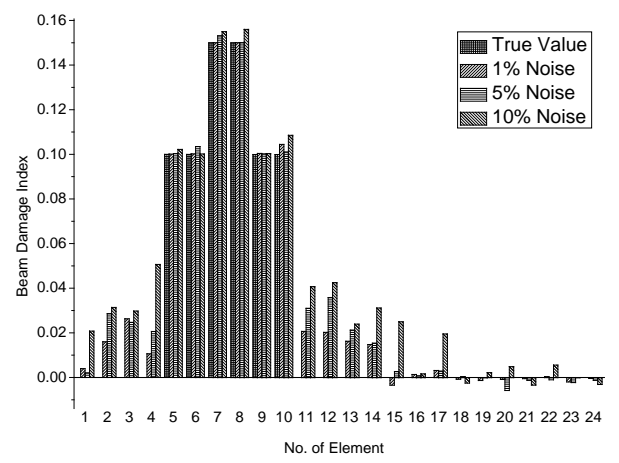

a) Beam damage index

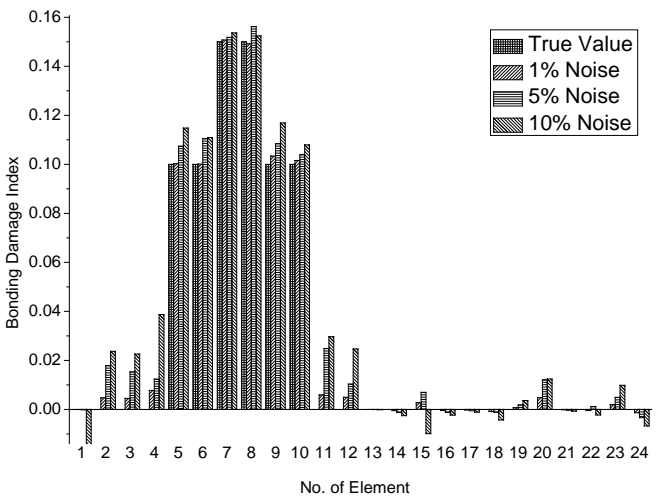

b) Bonding damage index

Figure 14 Identified beam and bonding damage in multiple elements with 11 sensors

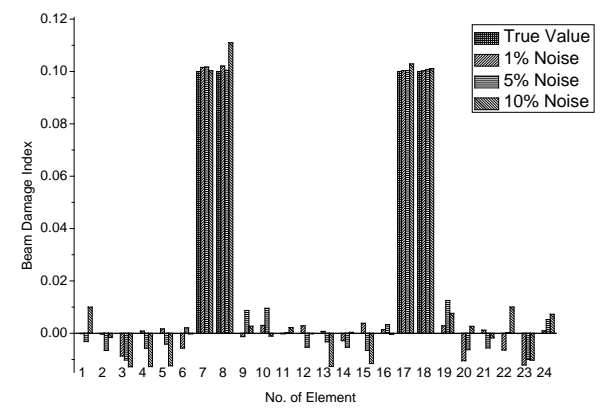

a) Beam damage index

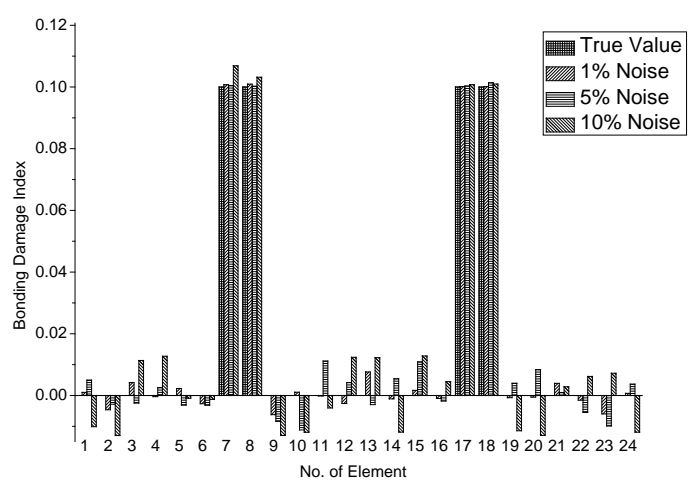

b) Bonding damage index

Figure 15 Identified beam and bonding damage in two separate elements with 11 sensors

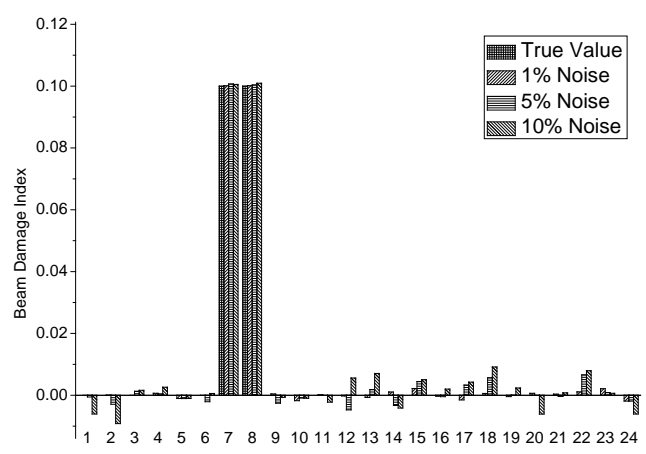

No. of Element

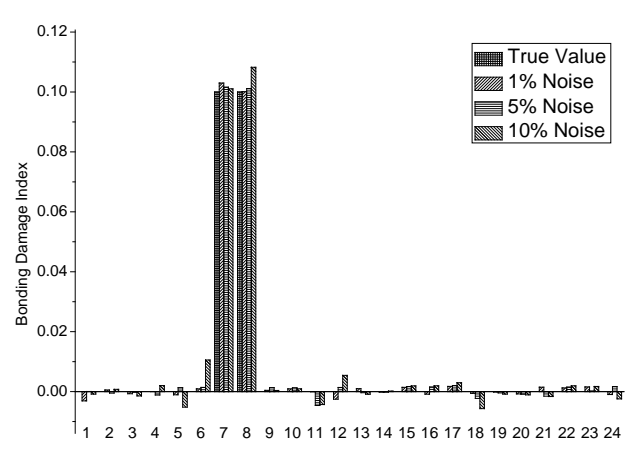

No. of Element

b) Bonding damage index

Figure 16 Identified beam and bonding damage in a single element with 23 sensors 


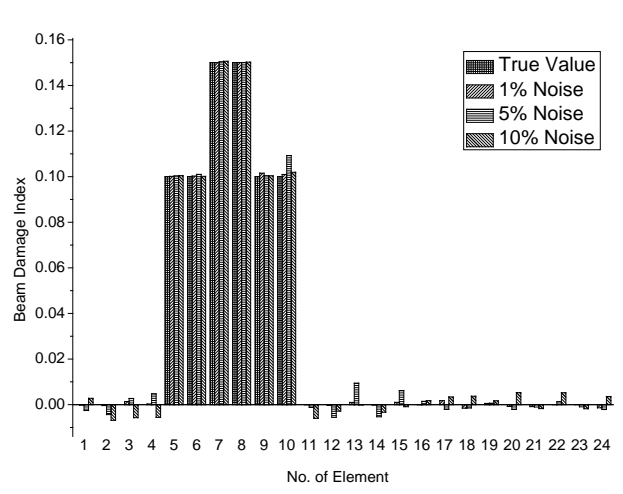

a) Beam damage index

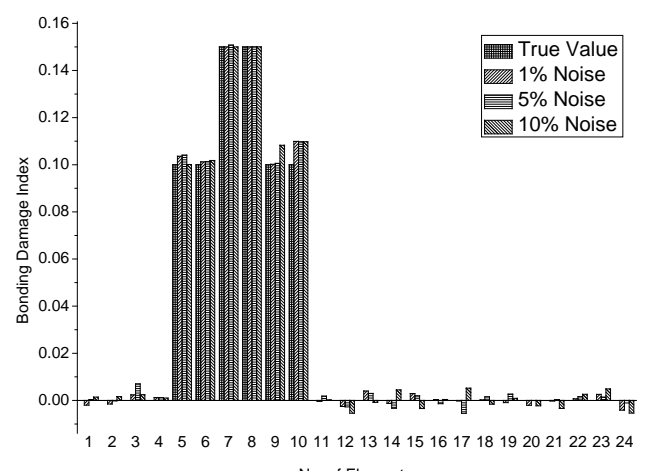

No. of Element

b) Bonding damage index

Figure 17 Identified beam and bonding damage in multiple elements with 23 sensors

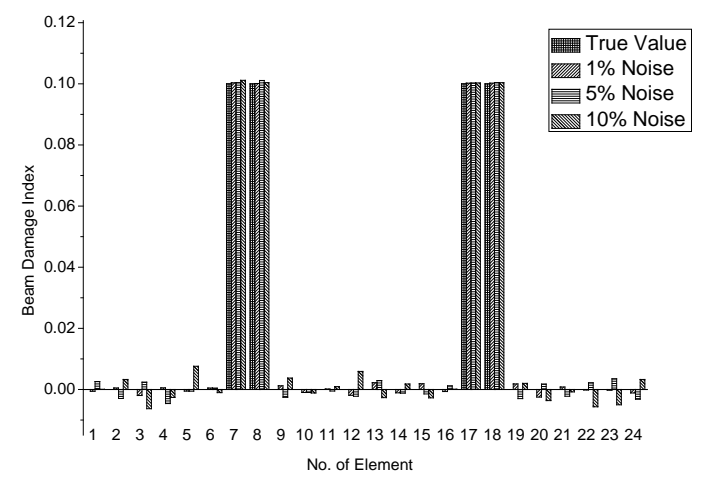

a) Beam damage index

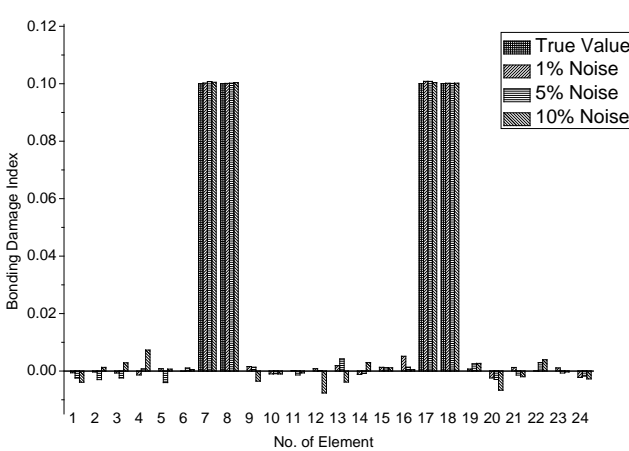

b) Bonding damage index

Figure 18 Identified beam and bonding damage in two separate elements with 23 sensors 